\title{
Differenz
}

Revista internacional de estudios heideggerianos y sus derivas contemporáneas

AÑO 7, NÚMERO 6: JULIO DE 2020. ISSN 2386-4877 - DOI: 10.12795/Differenz.2020.i06.07

[pp. 139-154]

Recibido: 07/06/2020

Aceptado: $21 / 06 / 2020$

\section{El concepto de tiempo de Hegel y Schelling en los Seminarios de 1927-1928 de Heidegger.}

\section{The concept of Hegel and Schelling's time on Heidegger's Seminars of 1927-1928.}

Luis Ángel Lome Hurtado

Universidad Michoacana de San Nicolás de Hidalgo

Resumen:

El objetivo es mostrar la interpretación del concepto de tiempo de Hegel y Schelling en los seminarios de 1927-1928 de Martin Heidegger. Primero se explica la confrontación que Heidegger realiza de Hegel antes y después de Ser y tiempo. Después, se analiza la primera confrontación con Schelling en 1927-1928. Y al final se concluye con los elementos centrales que el joven Heidegger retoma del concepto de tiempo de Hegel y Schelling.

Palabras clave: Temporeidad; Devenir; Desaparecer; Eternidad; Absoluto. 


\section{Abstract:}

The aim is to show the process of Hegel and Schelling's interpretation of concept of time on Martin Heidegger's seminars of 1927-1928. First, I explain the Heidegger's confrontation of Hegel before and after of Being and Time. Then, is analyzed the first Heidegger's confrontation with Schelling in 1927-1928. At the end, is concludes with the basics elements that the young Heidegger takes up from Hegel-Schelling's concept of time.

Keywords: Temporality; Becoming; Disappear; Eternity; Absolute.

\section{Introducción.}

Dentro del pensar de Martin Heidegger el problema del tiempo se circunscribe al interior del conflicto entre la ontología fundamamental y la tradición de la metafísica occidental, el cual alcanza su más alta exposición en el tratado Ser y tiempo publicado en abril de 1927. En este tratado fundamental se visualizan tres sentidos del tiempo: tiempo vulgar (vulgäre Zeit), temporeidad (Zeitlichkeit), temporaneidad (Temporalität). Esta división es la llave hermenéutica para entender la reflexión sobre el concepto de tiempo. La aparición del tratado fundamental consistió en la publicación de las dos primeras secciones de la "Primera parte": "Primera sección. Etapa preparatoria del análisis fundamental del Dasein" que realiza la interpretación analítica del Dasein y "Segunda sección. Dasein y temporeidad" que interpreta temporalmente la estructura del Dasein. Pero la "Tercera sección. Tiempo y ser" que proyectaba el horizonte trascendental del tiempo del ser (temporaneidad) y la "Segunda parte" del mismo no se publicaron; pero, según FriedrichWilhelm von Herrmann, la reelaboración de la Tercera sección se encuentra vertida en las lecciones del semestre de verano de 1927 llamadas Los problemas fundamentales de la fenomenología. Simultáneamente a esas lecciones Heidegger dictó un seminario bajo el nombre Aristoteles-Hegel-Seminar SS 1927 y posteriormente en el semestre de invierno dictó el seminario Schelling. Über das Wesen der menschlichen Freiheit WS 192728 (ambos aparecidos en GA 86 Seminare Hegel-Schelling en el 2011).

La propuesta de este escrito es mostrar que en esos seminarios acerca de Hegel y Schelling hay un considerable interés por la comprensión del concepto de tiempo propio del Idealismo alemán, el cual acontece a modo de confrontación, y su resultado es que Heidegger se apropia de ese tiempo para su pensar acerca del ser originario como temporaneidad, el cual determinará el posterior meditar del ser (Seyn). 


\section{Desaparecer.}

Desde Platón hasta Nietzsche se concibió el tiempo a partir del número, del cálculo, de la subjetividad, de la objetividad, de la afección y de la eternidad. Este tiempo es el propio de la experiencia metafísica del ser, al cual se opone el pensar ontológico de Heidegger. Pero esta oposición no se muestra como un rechazo definitivo de la historia del tiempo debido a que Heidegger logra reconocer en ella ciertos elementos que encauzan hacia una nueva gestación del tiempo como temporeidad. Este tiempo propio de la existencia humana recoge el carácter extático de Aristóteles, introduce el sentido kairológico del tiempo del cristianismo, se inspira por el temple de ánimo de Agustín y parte del flujo de la conciencia de Husserl. Pero, la relación de Heidegger con la constelación del Idealismo alemán (Kant, Schelling, Hegel) es especial y de sumo cuidado. El concepto kantiano de tiempo influyó en la génesis y estructura de Ser y tiempo. La filosofía de Schelling no corrió con la misma suerte durante ese período, sus referencias e interpretaciones son nulas. Y con Hegel, se encuentran diversos análisis del concepto de tiempo que reflejan incertidumbre y ambigüedad por parte del joven Heidegger ${ }^{1}$.

La asimilación heideggeriana de los textos hegelianos es difícil debido a la complejidad y oscuridad que contiene cada proposición especulativa insertada en el sistema del Idealismo absoluto. Heidegger padece de la ambigüedad filosófica que representa el lenguaje especulativo de Hegel, por una parte, afirma que el concepto de tiempo de Hegel es la figura más perfecta del tiempo de la tradición metafísica; y por otra parte, reconoce que dicho tiempo está muy próximo a una consideración del sentido auténtico del tiempo y, con ello, al desocultamiento del tiempo de la ontología.

El carácter especulativo del pensamiento filosófico alemán muestra que la influencia que ejerce Hegel sobre el joven Heidegger no es del todo clara, evidente, ni comprensible. La época de Ser y tiempo considera que la Ciencia de la lógica de Hegel representa la cima de la ontología tradicional y, por ende, el concepto de tiempo hegeliano se presenta como la síntesis de todas las determinaciones lógicas e históricas de la concepción filosófica del tiempo occidental. Parafraseando la reformulación kantiana que expresó alguna vez Ludwig Landgrebe, desde Aristóteles hasta Hegel el concepto de tiempo no dio un paso más adelante sino hasta la propuesta heideggeriana de la temporeidad e historicidad

1 En El concepto de tiempo (1924) y los Prolegómenos para una historia del concepto de tiempo (1925), Heidegger omite a Hegel, sobre esas lecciones de 1925 véase la siguiente referencia: "La segunda parte, 'Historia del concepto de tiempo' habría que pasar por tres estadios: Bergson, Kant y Newton, y Aristóteles (es visible el 'olvido' de Hegel)". PeÑAlVER, P., Del espíritu al tiempo. Lecturas de "El Ser y el Tiempo" de Heidegger. Barcelona, Anthropos, 1983, p. 60. La exposición del concepto de tiempo de Hegel se encuentra en las lecciones de invierno Lógica. La pregunta por la verdad (1925-1926) y en los últimos parágrafos de Ser y tiempo (1927) 
del Dasein². Y esto fue posible a partir de la apropiación del tiempo husserliano (tiempo del mundo y tiempo de la conciencia) de las Investigaciones lógicas, el cual Heidegger transformó en la contraposición ontológica de tiempo vulgar y tiempo auténtico. Bajo este proceder fenomenológico, Heidegger analiza el concepto de tiempo de Hegel.

Heidegger observa que Hegel toma plenamente su concepto de tiempo de la Física de Aristóteles. Por esta razón, en los parágrafos 20 y 21 de las lecciones de invierno Lógica. La pregunta por la verdad de 1925-1926, analiza la significación de tiempo desde la "Filosofía de la naturaleza" de la Enciclopedia de las Ciencias Filosóficas. En ese análisis se realiza una aproximación histórica acerca del problema ontológico del espacio-tiempo dentro del horizonte de la negatividad, esto llama la atención del joven Heidegger. Pero, a causa de la construcción metafísica del tiempo desde el espacio, la puntualidad y la naturaleza, Heidegger se separa de la ontología hegeliana. En los posteriores parágrafos de dichas lecciones Heidegger retorna a Kant y divisa con nuevos ojos el tiempo interno de la conciencia de la Crítica de la razón pura, en donde se observa un alejamiento del tiempo de la naturaleza y se entreabre una constitución del tiempo desde la subjetividad. Franco Volpi expresó que ese alejamiento de Hegel y Aristóteles se debe a la proximidad kantiana entre la subjetividad y la temporalidad, entre el tiempo y el ser; el resultado fue la introducción de ese tiempo trascendental transformado en la dinámica de la estructura del cuidado (Sorge) de la existencia del Dasein ${ }^{3}$.

Más allá de la constitución naturalista del tiempo, Heidegger siente admiración por el concepto de devenir de Hegel. En esas lecciones invernales de 1925-1926 se observa un interés por comprender la significación del fenómeno del devenir hegeliano, por esta razón expresa que:

El devenir que constituye el concepto de tiempo Hegel lo entiende como desaparecer, como la "abstracción del consumir" (§258 Añadido): el puro hacer desaparecer que desaparece ${ }^{4}$.

En estas líneas se percibe asombro y fascinación por el devenir que influye sobre el tiempo y el ser, pero también existe un sobresalto por el desaparecer del tiempo. Precisamente, me parece que este fue uno de los resultados más sobresalientes que obtuvo el joven Heidegger del sistema definitivo del Idealismo de Hegel, el devenir como un desaparecer que desaparece, el cual sólo deja la puerta abierta para el acceso del absoluto en tanto saber de lo absoluto como superación de la finitud.

2 Cfr. LANdgrebe, L. Fenomenología e historia. Caracas, Monte Ávila, 1975, p. 223.

3 Cfr. VolPI, F. Heidegger y Aristóteles. México D. F., Fondo de Cultura Económica, 2011, p. 116.

4 HeIDEgGeR, M. Lógica. La pregunta por la verdad. Madrid, Alianza, 2004, p. 208. 
Este concepto conductor del devenir como desaparecer forjó el destino del concepto de tiempo del Idealismo absoluto de Hegel dentro de la filosofía de la finitud de Heidegger. Debido a este suceso, la hermenéutica del sentido del ser como devenir y del tiempo como desaparecer es dejada a un lado por Heidegger; esto aunado a la experiencia vertiginosa que despertó la lectura de Hegel en el joven Heidegger, la cual provocó asombro y se expresó a través de palabras obscuras cuando dice:

(...) por qué, si el tiempo es devenir, tiene que ser precisamente desaparecer. Ya Aristóteles, en su explicación del tiempo, imputó el tiempo al devenir y no al surgir. Es oscuro por qué esto es así,

y cuando se expresan palabras que reflejan un total desacuerdo: "Hegel puede decirlo todo sobre cualquier cosa. $Y$ hay gente que en semejante confusión descubren un pensamiento profundo"6 y también cuando se expresa más prudentemente al decir:

Desde la base hegeliana sigue siendo incomprensible por qué el tiempo es, como él dice, consumir. En el horizonte de la dialéctica hegeliana, este fenómeno que ya la experiencia vulgar del tiempo experimenta inmediatamente tiene que seguir siendo un enigma?

Estas aseveraciones muestran la distancia que puso Heidegger frente a Hegel, sin embargo expresan también el carácter contradictorio de la interpretación del joven Heidegger sobre el maestro de lo absoluto. Pero en su conjunto, estas declaraciones son el testimonio de la fuerte influencia que posee el Idealismo hegeliano al interior de la ontología fundamental del joven Heidegger. ¿Acaso será que durante esta época el pensamiento que tiene Heidegger de Hegel no se encuentra suficientemente preparado para su confrontación filosófica? En lo siguiente se muestra cómo fue abordado el concepto de tiempo de Hegel en Ser y tiempo.

\section{Devenir intuido.}

En el penúltimo parágrafo ( $§ 82$ ) de Ser y tiempo se ejecuta una confrontación con el concepto de tiempo de Hegel, el cual es determinado a partir de la relación entre espíritu y tiempo. Heidegger indica que el tiempo hegeliano es la realización conceptual más radical de la comprensión vulgar del tiempo, la razón se encuentra en que a través de la dialéctica y de la negatividad el tiempo es conducido hacia el concepto y encuentra su determinación

\footnotetext{
$5 \mathrm{lb}$.

6 lb. p. 209.

7 lb. p. 210
} 
más cabal como "devenir intuido"8. Esta exposición se caracteriza principalmente a partir de la acuñación del devenir no como un mero desaparecer, sino como el ahora que se mantiene constantemente como presente; por esta razón, el tiempo se entiende desde el presente como dimensión preeminente del devenir. El devenir intuido del tiempo en su constante desaparecer conduce hacia el pensamiento del presente como eternidad.

En esa confrontación del § 82 del tratado fundamental, Heidegger menciona ahora que el tiempo hegeliano es visto como "abstracción de la consunción", esto significa que la dimensión del tiempo no es tomada por Hegel desde el desaparecer o consumir de la vivencia cotidiana del tiempo, sino que el tiempo se separa de esa experiencia y alcanza el momento de la abstracción lógica como "negación de la negación (es decir, de la puntualidad)"10 de la conciencia del tiempo. El enfásis de la formulación "devenir intuido" no recae en el "devenir" como "pasar", sino en la "intuición" que otorga al estado de llegar a ser de una cosa el ser "algo absolutamente abstrato, ideal"11.

La idealidad del devenir permite que el espíritu como absoluto saber de sí pueda caer dentro del tiempo. Al espíritu le corresponde su autorevelación (Selbstoffenbarung) y su realización (Verwirklichung) constante en la historia del mundo ${ }^{12}$. El espíritu es negación de la negación, absoluta; el tiempo es negación de la negación, inmediata. El espíritu es negatividad absoluta; el tiempo es negatividad abstracta. El espíritu es concepto que se sabe a sí mismo; el tiempo es concepto solamente intuido, es decir, es el sí mismo puro externo intuido. El espíritu es concepto puro; el tiempo es intuición vacía. Cuando se revela el concepto puro en el tiempo, acontece el momento de la negatividad del absoluto, y esto conlleva a que el absoluto suprime el tiempo: la eternidad es presente.

Esta confrontación con el tiempo hegeliano refleja la madurez hermenéutica de Heidegger para abordar y proyectar cuestionamientos para la pregunta conductora de su investigación. Esto se acentúa cabalmente por las conclusiones a las que llega en su exposición, las cuales se expresan al final del parágrafo § 82 cuando dice que la correlación negativa de tiempo-espíritu encubre y deja en la oscuridad la "caída" y la "realización" del

8 HEIDEgGER, M. Ser y tiempo. Madrid, Trotta, 2006, p. 444.

9 lb. p. 445.

$10 \mathrm{lb}$.

11 lb. p. 444.

12 Las palabras alemanas Selbstoffenbarung y Verwirklichung son traducidas por J. E. Rivera (HEIDEGGER, M. Ser y tiempo. Op. cit. p. 447) como "automanifestación" y "actualización"; mientras que J. Gaos (Heidegger M. El ser y el tiempo, México D.F., Fondo de Cultura Económica, 2002, p. 467) las traduce como "autorevelación" y "realización". De acuerdo con el proyecto filosófico del Idealismo alemán, de donde surgen Hegel y Schelling, me parece más adecuado seguir aquí la versión de J. Gaos. 
espíritu dentro del tiempo; pero el más eminente resultado que se obtiene consiste en que Hegel no explica el origen del tiempo mismo. El último parágrafo (§ 83) de Ser y tiempo proyecta la necesidad de clarificar el surgir y el origen del tiempo del mundo y del tiempo de la vida, esto desde el preguntar acerca de las condiciones de posibilidad para hacer ver que el tiempo como temporeidad posee una base originaria, es decir, de aquello que hace posible que el tiempo se temporice como temporeidad: la temporaneidad (Temporalität) como horizonte trascendental del ser en general.

Interesante es que en los últimos parágrafos de Ser y tiempo se haya realizado una de las interpretaciones más radicales acerca del tiempo, y que para obtener dichas conclusiones se haya construido una confrontación directa y puntual con la filosofía del Idealismo alemán de Hegel. La "Segunda Sección" del tratado de Heidegger recibe un impulso eficaz que será desarrollado en la "Tercera Sección. Tiempo y ser" que aparece en las lecciones del semestre de verano de 1927 Los problemas fundamentales de la fenomenología. Allí, se desarrolla más contundentemente el problema del tiempo como temporaneidad, es decir, del tiempo del ser propio y exclusivo de esa época de Heidegger ${ }^{13}$. Pero ahí, las referencias al concepto de tiempo de Hegel son escasas. Esto nos lleva a la consideración de que Heidegger ha sobrepasado la dimensión tradicional del tiempo de Hegel.

En el seminario Aristoteles-Hegel Seminar SS 1927 aparecen referencias al concepto de tiempo de Kant en los apartados 8, Kant, Zeit y 9, Kant, Zeit. Lo que se enfatiza en ellos es el dominio del concepto formal-mundano del tiempo kantiano y su imposibilidad para pasar a una nueva comprensión del tiempo. Bajo esta directriz lógica, Heidegger coloca en la nota 70, Hegel, Logik la pertenencia de la definición hegeliana del tiempo a la concepción lógica metafísica del ser:

Pero esto es negación de la negación -es decir, "es"- como devenir puro (Tiempo -el devenir intuido).

El "devenir" comienza -es decir, "deviene" y eso expresa- "es" la verdad del ser y la nada ${ }^{14}$.

13 Es importante mencionar que sólo durante la época del tratado Ser y tiempo se establece la temporaneidad (Temporalität) como el tiempo del ser en general. Cuando Heidegger explora el tiempo del ser como evento (Ereignis) alrededor de 1936, se puede ver que sólo nombra "el tiempo del ser" (die Zeit des Seins) (HeIDEgGer, M. Aportes a la filosofía. Acerca del evento. Buenos Aires, Biblos, 2006, p. 400), dejando la temporaneidad como un tema de un proyecto ya transformado. Desde aquí es conveniente revisar la interpretación que hace GoMEZ PEDRIDO, M. "La interpretación de Heidegger del devenir eterno en la filosofía de Schelling", en Cuadernos de Filosofía. № 67-68, 2017.

14 "Dieses aber ist Negation der Negation -d.h. es "ist"- als reines Werden. (Zeit -das angeschaute Werden.) / Das "Werden" beginnt -d.h. es "wird" und das sagt- es "ist" die Wahrheit von Sein und 
El tiempo como "devenir intuido" es nombrado "devenir puro" y "verdad del ser y la nada". En este sentido, se observa que la interpretación del tiempo hegeliano se traslada hacia la exégesis de los conceptos puros del ser desarrollados por Hegel en su "Doctrina del ser" de la Ciencia de la Lógica. En ese lugar se expresa que el ser y la nada son lo inmediato indeterminado, esto es lo que escapa al discurso lógico, y que su superación es el devenir como consumación y traspaso hacia el inicio de las determinaciones del pensamiento. Heidegger al colocar la correlación entre el concepto de tiempo y el devenir se inclina hacia el análisis de este último para encontrar la constitución del concepto de tiempo de Hegel. En la nota 20, Diferencia de la primera y la segunda tríada y el comienzo (Unterschied der Ersten und Zweiten Triade und der Anfang) Heidegger piensa acerca del comienzo como "ser y nada" lo siguiente:

Enigma - ¡iCómo Hegel llega al devenir!! No sólo un aparecer. -

Llegar a algo -sin que el devenir esté primero ahí- llegar al devenir -ien la esencia del devenir!

ilmposible! ${ }^{15}$.

Si el tiempo es devenir, si éste encuentra su esencia en la dialéctica y en la negatividad del ser puro y la nada pura, entonces el tiempo descubre su esencia y su determinación con la clarificación del devenir; pero en estas operaciones puras del pensamiento, el devenir es un enigma (Rätsel) y Hegel no da las indicaciones lógicas acerca de cómo es que se obtiene el devenir, el cual no es únicamente un mero aparecer (Schein), sino algo que ya está presente para que la existencia y las determinaciones del ser puedan realizarse ${ }^{16}$. Heidegger acentúa que el devenir tiene que llegar a ser sin que él mismo se encuentre ahí, y muestra que dentro de la lógica-ontológica del ser hegeliano, es imposible discursivamente pensar en la esencia del devenir:

Nicht". HeIDEgGER, M. Seminare Hegel-Schelling. GA 86. Frankfurt am Main, Vittorio Klostermann, 2011, p. 41).

15 "Rätsel - wie Hegel zum Werden kommt!! / Nur ein Schein. - / Kommen zu etwas -ohne dass Werden erts da ist- Kommen zum Werden -in das Wesen des Werdens! / Unmöglich!" (Ib., p. 13).

16 Cfr. El carácter no pensado del tiempo y el devenir de Hegel en FeRnández, J. "Cuestiones litigantes. El diálogo de Heidegger con Hegel y Schelling" en Differenz. Revista internacional de estudios heideggerianos y sus derivas contempóraneas. № 5. 2019, p. 188. 
El devenir -como lo que ha salido del ser y de la nada, es lo primero que está deviniendo (lógicamente la primera verdad- el primer ente, pero así como resultado inmediato, todavía no verdadero) $)^{17}$.

En el comienzo (Anfang) el ser puro y la nada pura obtienen su primera verdad del devenir, pero Heidegger enfatiza que: "el devenir no deviene primero -sino que sólo permite degradarse a sí en el ser- él mismo no tiene la fuerza -para llegar al devenircuando éste ya no es"18. Esto significa que el devenir se presenta primero como la primera determinación lógica del ser, pero su propio surgir y su propio tiempo permanecen indeterminados y en la oscuridad.

En la nota 66 del Aristoteles-Hegel-Seminar, Heidegger escribe una serie de frases que intentan pensar la relación entre el concepto hegeliano de tiempo como devenir y el tiempo del ser (temporaneidad). A continuación cito la nota:

66. Devenir como concepto (verdad originaria) del ser y temporaneidad. Temporaneidad y negación

Devenir mismo - como cambio (saltar de - a). El "de" y el "a" y iel transformar!

La apariencia de esa muy óntica interpretación del ser se supera a través del descubrimiento del horizonte originario para la comprensión del ser.

La ley relativa a la temporaneidad hace comprensible la causa de que el devenir es la verdad del ser.

Al mismo tiempo es evidente - como la Lógica de Hegel es una antigua insuficiencia condicionada- aunque orienta la historia en la sustancia.

Acontecer-temporaneidad-devenir.

Sur-gir y perecer:

a) un originario sentido de la temporeidad.

b) aún más radical como temporaneidad.

Temporaneidad de la "mediación" y sus categorías (ERDMANN, § 88 ss).

17 "Das Werden -als hervorgegangen aus Sein und Nichts ist das erste Gewordene (logisch die erste Wahrheit -das erste Seiende- aber so als unmittelbares Resultat -noch wieder unwahr)". HEIDEGGER, M. Seminare Hegel-Schelling. Op. cit., p. 17.

18 "Das 'Werden' wird nicht erst -sondern lässt sich nur degradieren zum Sein- das selbst nicht die Kraft hat -zum Werden zu kommen- wenn dieses nicht schon ist". Ib., p. 37. 
¡Posibilidad de la mediación! ¡Principio de la relación!

Referencia como categoría formal ontológica - ¡Relación como categoría

formal apofántica!

Temporaneidad del pensamiento y verdad ${ }^{19}$.

En esta nota, primero se delimita la significación del devenir como un cambio repentino (Umschlag), como un saltar (übergehen) de un estado a otro y, luego, como la transformación (über-gehen) de ese estado. Aquí, Heidegger enmarca el devenir puro de Hegel como una interpretación óntica del ser (percepción del cambio), la cual concuerda con Aristóteles, y únicamente puede ser superada a partir de una posición ontológica del devenir proporcionada por la temporaneidad como horizonte trascendental del ser en general. Este tiempo, que no obtiene su ser a partir de la percepción y contabilización del cambio, sino del surgir mismo, se presenta como la base horizontal del devenir hegeliano (devenir como verdad del ser) y es la condición de posibilidad de la negatividad como mediación de todas las relaciones del pensamiento ${ }^{20}$. Aquí, Heidegger crítica el pasar, el transformar, el desaparecer y la negatividad de la concepción del tiempo como devenir intuido de Hegel. A pesar de que dicho tiempo haya sido la orientación de la concepción de la historia, el resultado es que la temporaneidad heideggeriana se presenta como devenir y acontecer y, con ello, piensa en el "surgir" y el "perecer" del tiempo, como fenómenos temporales que no están presentes en Hegel. Este surgir y perecer son la constante peculiaridad del acontecer temporal del ser heideggeriano, y únicamente desde ellas es como se puede pensar en el tiempo del Dasein (temporeidad) y el tiempo del ser (temporaneidad).

19 "66. Werden als Begriff (eigentliche Wahrheit) des Seins und Temporalität. Temporalität und Negation. / Werden selbst - als Umschlag (Übergehen von - zu). / Das "von" und das "zu" und das Über-gehen! / Die Äusserlichkeit dieser mehr ontische Interpretation des Seins überwinden durch Aufdenkung des ursprünglichen Horizontes für das Seinsverständnis. / Aus Temporalität das relative Recht verständlich machen, weshalb Werden die Wahrheit des Seins. / Zugleich daraus ersichtlich -wie Hegels Logik eine antik bedingte Halbheit- obzwar in der Substanz auf Geschichte orientiert. / Geschehen und Temporalität und Werden. / Ent-stehen und Vergehen: / a) ein ursprünglicher Sinn der Zeitlichkeit / b) noch radikaler als Temporalität. / Temporalität der "Vermittlung" und ihrer Kategorien (ErDmann § 88 ff.). / Möglichkeit der Vermittlung! Grundsätzlich der Beziehung! / Bezug als formale ontologische Kategorie - Beziehung als formale apophantische Kategorie! / Temporalität der Gedachtheit und Wahrheit". Ib., pp. 38-39.

$20 \mathrm{Cfr}$. "En definitiva, Hegel está sobre la pista de una gran verdad cuando dijo que el ser y la nada son idénticos, esto es, se pertenecen mutuamente. La pregunta más radical es sin duda: ¿Qué es lo que hace posible semejante copertenencia originaria?". HEIDEGGER, M. Los problemas fundamentales de la fenomenología. Madrid, Trotta, 2000, p. 371. 
Desde este punto, el horizonte originario para la comprensión del ser deberá captar un sentido ontológico del devenir como acontecer y tiempo originario, incluso tiene que contener esa explicación del surgir del tiempo mismo para poder ser la base trascendental del tiempo del mundo y del tiempo de la vida. Por esta razón, durante la confrontación con el maestro del absoluto, Heidegger se ha apropiado ya del tiempo a través del devenir como desaparecer (degradación) y, con ello, busca pensar no el pasar del tiempo, sino su surgir o emerger. En ese mismo año de 1927, después del Aristoteles-Hegel-Seminare, Heidegger se confronta con Schelling.

\section{Devenir-revelado.}

En su época universitaria y a través de su maestro Carl Braig, Heidegger conoció la teología especulativa de Schelling y Hegel. Pero, su primer contacto con el tratado Investigaciones filosóficas sobre la esencia de la libertad humana y los objetos con ella relacionados (1809), (Freiheitsschrift), se lo debe a Karl Jaspers, de quien recibió en los primeros meses de 1926 una obra de Schelling. Heidegger quedó fascinado e impregnado de la potencia discursiva del Freiheitsschrift, a tal punto que colocó inmediatamente a Schelling en el mismo nivel filosófico de $\mathrm{Hegel}^{21}$. Se puede decir que esta lectura marcó el pensar de Heidegger durante ese año en el que la "Introducción" y la "Primera sección" de la "Primera parte" de Ser y tiempo estaban en impresión, y en el que la "Segunda sección" del mismo se encontraba en proceso de redacción. Friedrich-Wilhelm von Herrmann relata que Heidegger sostuvo fructíferas conversaciones con Karl Jaspers a principios de enero de 1927, en donde revisaron los capítulos tercero y cuarto del tratado fundamental que abordan la temporeidad de la existencia del Dasein, y en donde posiblemente se discurrió acerca del proyecto del sentido de la temporaneidad como horizonte trascendental del ser, el cual sería desarrollado en la "Tercera sección. Tiempo y ser" como "Segunda parte" de

21 Schwab y Schwenzfeuer mencionan que el proyecto del seminario de 1927/28 fue motivado por la correspondencia entre Heidegger y Karl Jaspers en 1926. En la carta del 24 de abril de 1926 Heidegger le comunica a Jaspers su fascinación por el tratado de Schelling, a quien coloca en el mismo nivel de Hegel. Cfr. SCHWAB, Ph.; Schwenzfeuer, S., "Protokolle einer Übung von Martin Heidegger zu Schellings Abhandlung über das Wesen der menschlichen Freiheit aus dem Wintersemester 1927/28 in Marburg", en HüHN, L.; JANTZEN, J. (Hg.). Heideggers Schelling - Seminar (1927/28). Die Protokolle von Martin Heideggers Seminare zu Schellings "Freiheisschrift" (1927/28) und die Akten des Internationalen Schelling-Tags 2006. Stuttgart, Bad-Cannstatt, 2011. pp. 289290. Así mismo, para una idea general de la recepción de Schelling en Heidegger véase: RIVERA DE RosALES, J. "La recepción de Schelling en Heidegger", en Pensamiento. Revista de investigación e información filosófica, vol. 70, no 262, 2014, pp. 197-202. 
Ser y tiempo, que aparece posteriormente en las lecciones Los problemas fundamentales de la fenomenología de ese mismo año ${ }^{22}$.

Después de esas lecciones de verano, Heidegger dictó durante el semestre de invierno de 1927-1928 el seminario Schelling. Über das Wesen der menschlichen Freiheit. WS $1927 / 28$, el cual es considerado por Lore Hühn, Philipp Schwab y Sebastian Schwenzfeuer como la primera confrontación con el Freiheitsschrift de Schelling ${ }^{23}$. Además, en el Seminare Hegel-Schelling (GA 86) aparece dicho seminario junto con tres protocolos realizados por los participantes del mismo (Werner Bohlsen, Wolfgang-Günter Friedrich, Elisabeth Krumsiek).

Del seminario sobre el Freiheitsschrift se conservan doce breves notas acerca de esa primera confrontación con Schelling, en ellas no se habla directamente sobre el concepto de tiempo de este pensador, ni acerca del tiempo de la tradición filosófica; pero se esquematizan diversos temas centrales que fueron desarrollados al interior de seminario, entre los cuales sale a relucir el problema del ser originario (Urseyn) como devenir ${ }^{24}$. En las notas se muestra la fascinación de Heidegger por la lectura del Freiheitsschrift, en ellas aparece una transcripción de la frase de Schelling que dice: "El ser sólo deviene a sí sensible en el devenir (403)"25. Schelling piensa el ser (Wesen) como fundamento (Grund) y existencia (Existenz), estos principios eternos son simultáneos e inseparables, pero tienen dualidad y oposición. Werner Bohlsen atestiguó en su protocolo fechado el 7 de diciembre de 1927 que Heidegger no interpretó el ser (Wesen) desde los conceptos latinos de essentia, realitas, ni desde el término kantiano de realidad (Wirklichkeit), sino desde un sentido ontológico que conecta el ser de Schelling con la antigua ontología griega del ser (ti estin); lo mismo pasa con la existencia (Existenz), la cual no proviene del término latino existentia, sino que mienta el constante efectuarse (Vollzug) del ser mismo ${ }^{26}$. Esto muestra que el ser de Schelling no fue inmediatamente identificado a partir de los

22 Cfr. von Herrmann, F.-W. La segunda mitad de Ser y tiempo. Sobre los conceptos fundamentales de la fenomenología de Heidegger. Madrid, Trotta, 1997, pp. 30-36.

23 Cfr. HüHN, L. "Heidegger-Schelling im philosophischen Zwiegespräch - Der Versuch einer Einleitung". En HüHN, L; JANTZEN, J. (Hg.). Op. cit, p. 3. Y también, SCHWAB, Ph.; SCHWENZFEUer, S. Op.,cit., p. 289.

24 En el año 1946 se editaron las primeras dos versiones de 1811 y 1813 de las Edades del mundo de Schelling, la última versión de 1815 se publicó en 1861. Si Heidegger conoció dicho texto tuvo que haber sido el de esta última versión, pero estoy de acuerdo con Jorge Eduardo Fernández cuando expresa que en la obra de Heidegger (Ser y tiempo) no se pueden encontrar fácilmente las referencias a las Edades del mundo de Schelling. Véase: FernÁNDEZ, J. Op. cit. p. 188.

25 "Das Sein wird sich nur im Werden empfindlich (403)". HEIDEGGER, M. Seminare Hegel-Schelling. GA. 86. Op. cit., p. 52.

26 Cfr. Ib. p. 529. 
conceptos fundamentales de la tradición metafísica, sino que la exégesis se desarrolló con cautela frente a la revelación de nuevos conceptos especulativos y totalmente opuestos a la filosofía de Hegel.

En el seminario Schelling. Über das Wesen der menschlichen Freiheit. WS 1927/28 hay una anotación de Heidegger que dice: "Devenir - Revelado"27. Esta indicación tiene interés debido a que el ser originario (Urseyn) es el infundamento (Ungrund) como la absoluta indiferencia del fundamento y existencia. El infundamento tiene que manifestarse en sus principios eternos, esto es, se revela en ellos como un movimiento de sí mismo. El ser como infundamento es lo absoluto que deviene en sus principios eternos. Werner Bohlsen acentuó que dicho absoluto no precede al tiempo (kein Vorhergehen der Zeit), ni sostiene una prioridad de la esencia (Priorität des Wesens) ${ }^{28}$. Y en su protocolo del 21 de diciembre de 1927, Wolfgang-Günter Friedrich expresó que el tratamiento ontológico no concibió al infundamento como la nada pura, ni como un mero absurdo, sino como el devenir que desaparece en sus principios eternos y simultáneamente esencia en ellos ${ }^{29}$. Por esta razón, un cuestionamiento transversal presente en los tres protocolos fue la manera en cómo se puede pensar un absoluto (Absolute) que deviene en la existencia, o dicho en otras palabras, cómo se concibe una eternidad desde el plano de la existencia temporal. El devenir-revelado posee la característica de ser un absoluto que debe de devenir para ser él mismo.

Desde la lectura en 1926 del Freiheitsschrift, se abrió para Heidegger una nueva perspectiva de la eternidad y del devenir a partir de la articulación de un devenir eterno o una eternidad que está deviendo. Esto se puede comprobar a través del protocolo de Elisabeth Krumsiek datado el 8 de enero de 1928 en donde se describe el tratamiento que se realizó en el seminario acerca del concepto de tiempo de Schelling: el tiempo inicia en la primera creación (perspectiva teológica), el tiempo es concebido como el proceso de un proseguir (perspectiva naturalista), y el tiempo es subjetivo (perspectiva kantiana). Para Heidegger estas características encasillaron el concepto de tiempo de Schelling dentro del tiempo ordinario o vulgar. Pero Elisabeth Krumsiek habla de una extraña duplicidad (eigentümliche Doppelung): un tiempo de lo viviente (Zeit des Lebenden) y de un tiempo independiente del observador (Beobachter) ${ }^{30}$. Aquí se puede atisbar la posible atracción de Heidegger hacia Schelling porque el tiempo posee un estatuto ontológico en cuanto

27 "Offenbar-werden". Ib., p. 50.

28 Cfr. Ib., p. 529.

29 Cfr. Ib., p. 535

30 Cfr. Ib. pp.547-548. 
supera la subjetividad moderna, y en cuanto se presenta como algo que atraviesa la vida eternamente. La aporía de lo eterno y el devenir es formulada en el último protocolo así:

En ese "eterno" yace una nueva dificultad. Cuando en Schelling, particularmente en la referencia a la historia, el tiempo mantiene una íntima conexión con el devenir, pero el devenir como amor es concebido y el amor como el ser de Dios, entonces debe ser también un devenir en la eternidad, así que él no puede ser un nunc stans, sino algo otro. Aquí está de nuevo una pregunta abierta ${ }^{31}$.

El devenir en la eternidad abre una nueva perspectiva del tiempo. La eternidad no es el permanecer inmóvil (nunc stans) o el siempre (aei) ni lo atemporal o supratemporal (aeternitas) del discurso teológico-metafísico de la sustancia, sino que es aquello que encuentra su ser a través del devenir de la existencia ${ }^{32}$. ¿Hasta qué punto el devenir eterno provocó cierto silencio e incertidumbre en aquella aula de la universidad de Marburgo a finales de 1927 e inicios de 1928? Ciertamente, no se puede saber, las escasas notas de Heidegger no lo permiten; pero en las últimas líneas escritas por Elisabeth Krumsiek se deja captar la potencialidad ontológica del tiempo de Schelling. Esto nos lleva a pensar que Heidegger descubre en el devenir de Schelling aquello que no había alcanzado a obtener del devenir de Hegel, de esta manera, el devenir intuido como desaparecer del desaparecer de la dialéctica hegeliana colisiona con el devenir eterno del absoluto de Schelling. Se encuentra aquí una óptica alterna del tiempo, que implica no un tiempo que pasa o que desaparece, sino un tiempo que surge entre la eternidad y el devenir, y este "surgir" del tiempo es aquello que buscaba Heidegger desde 1925 y es lo que determinará su posterior pensar acerca del ser (Seyn) a partir de 1936 en su obra Aportes a la filosofía. Acerca del evento.

31 "In diesem 'ewig' liegt eine neue Schwierigkeit. Wenn bei Schelling, besonders in Bezug auf die Geschichte, die Zeit mit dem Werden in engem Zusammenhang steht, das Werden aber als Liebe gefasst wird und die Liebe als das Sein Gottes, dann muss auch in der Ewigkeit ein Werden sein, so dass sie nicht sein kann ein nunc stans, sondern etwas anderes. Hier ist wieder eine offene Frage". lb., p. 548.

$32 \mathrm{~A}$ partir de aquí se puede deducir un cambio radical para el tratamiento del concepto de tiempo en Heidegger, quien en la conferencia El concepto de tiempo (1924) ante la sociedad teológica de Marburgo había deslindado su tratamiento de la eternidad característica del lenguaje teológico. Véase HeIDEgGER, M. El concepto de tiempo. Madrid, Trotta, 2001, pp. 23-25. 


\section{Conclusión.}

Estas consideraciones han evidenciado que las concepciones de Hegel y Schelling también han contribuido para la configuración ontológica del tiempo en el joven Heidegger. Esto ha sido posible a partir de la publicación en 2011 de dichos seminarios de 1927 y 1928 contenidos en la GA 86. Seminare Hegel-Schelling. La intersección entre la ontología fenomenológica de Heidegger y los posicionamientos fundamentales del Idealismo alemán puede ser comprendida desde el problema filosófico del tiempo, del cual se ha intentado realizar una reconstrucción hermenéutica desde lo ya conocido o dado por supuesto de la interpretación heideggeriana de Hegel, y desde la inexistente influencia documentada del Schelling de Heidegger dentro de la época correspondiente a Ser y tiempo. Se puede decir que en esos años Heidegger comienza a edificar su interpretación colosal de la metafísica del Idealismo alemán, la cual a partir de 1936 será uno de los impulsos centrales de su pensamiento del ser (Seyn) como evento (Ereignis); pero esto, ya es otra historia, por ahora basta con lo anteriormente expuesto.

\section{Referencias bibliográficas.}

FERnÁNDEZ, J. "Cuestiones litigantes. El diálogo de Heidegger con Hegel y Schelling", en Differenz. Revista internacional de estudios heideggerianos y sus derivas contemporáneas. № 5, 2019.

Gomez PedRIDo, M. "La interpretación de Heidegger del devenir eterno en la filosofía de Schelling", en Cuadernos De Filosofía. № 67-68, 2017, pp. 49-68.

HEIDEGGER, M. Los problemas fundamentales de la fenomenología. Tr. J. J. García-Norro. Madrid, Trotta, 2000.

Heidegger, M. El concepto de tiempo. Tr. R. Gabás, J. Adrián Escudero. Madrid, Trotta, 2001.

Heidegger, M. El ser y el tiempo. Tr. J. Gaos. México D. F., Fondo de Cultura Económica, 2002.

Heidegger, M. Lógica. La pregunta por la verdad. Tr. A. Ciria. Madrid, Alianza, 2004.

HeIdegger, M. Ser y tiempo. Tr. J. E. Rivera. Madrid, Trotta, 2006.

Heidegger, M. Aportes a la filosofía. Acerca del evento. Tr. D. V. Picotti. Buenos Aires, Biblos, 2006. 
HEIDEGGER, M. Seminare Hegel-Schelling. GA 86. Frankfurt am Main, Vittorio Klostermann, 2011.

von HerrmanN, F.-W. La segunda mitad de "Ser y tiempo". Sobre los problemas fundamentales de la fenomenología de Heidegger. Tr. I. Borges-Duarte. Madrid, Trotta, 1997.

HÜHN, L. "Heidegger-Schelling im philosophischen Zwiegespräch - Der Versuch einer Einleitung", en HüHN, L.; JANTZEN, J. (Hg.). Heideggers Schelling - Seminar (1927/28). Die Protokolle von Martin Heideggers Seminar zu Schellings "Freiheitsschrift" (1927/28) und die Akten des Internationalen Schelling-Tags 2006. Stuttgart, Bad-Cannstatt, 2011, pp. 3-44.

LANDGREBE, L. Fenomenología e historia. Caracas, Monte Ávila, 1975.

PeÑAlVER, P. Del espíritu al tiempo. Lecturas de "El Ser y Tiempo" de Heidegger. Barcelona, Anthropos, 1989.

Rivera de Rosales, J. "La recepción de Schelling en Heidegger", en Pensamiento. Revista de investigación e información filosófica. Vol. 70. №. 262, 2014, pp. 197-202.

SCHWAB, Ph.; SCHWENZFEUER, S. "Protokolle einer Übung von Martin Heidegger zu Schellings Abhandlung über das Wesen der menschlichen Freiheit aus dem Wintersemester 1927/28 in Marburg" En HüHN, L.; JANTZEN, J. (Hg.). Heideggers Schelling - Seminar (1927/28). Die Protokolle von Martin Heideggers Seminar zu Schellings "Freiheitsschrift" (1927/28) und die Akten des Internationalen Schelling-Tags 2006. Stuttgart, Bad-Cannstatt, 2011, pp. 263-317.

VolPI, F. Heidegger y Aristóteles. Tr. M. J. de Ruschi. México D. F., Fondo de Cultura Económica, 2011. 